\title{
Identification of Mycobacterium bovis in patients diagnosed with pulmonary and extrapulmonary tuberculosis
}

\author{
Alejandro Hernández-Solís, ${ }^{*}$ Maribel González-Villa, ${ }^{2}$ Raúl Cícero-Sabido, ${ }^{1}$ \\ Heleodora González-González, ${ }^{1}$ Yesenia Colín-Muñoz, ${ }^{1}$ Alejandra Camerino-Guerrero ${ }^{1}$ and \\ Ernesto Ramírez-González²
}

${ }^{1}$ Secretaría de Salud, Hospital General de México "Dr. Eduardo Liceaga", Pulmonology Unit; '2Secretaría de Salud, Institute of Epidemiological Diagnosis and Reference. Mexico City, Mexico

\begin{abstract}
Introduction: In Mexico, there is an alarming increase in the number of cases of Mycobacterium bovis infection on pulmonary and extrapulmonary presentations. The lack of timely identification triggers complications and increases mortality. Objective: To know the frequency of $M$. bovis infections in clinical samples of patients with tuberculosis in the mycobacteria laboratory of a reference hospital in Mexico City. Method: Prospective, descriptive study. Strains isolated from biological material were studied in Löwestein-Jensen and MGITI960 cultures. M. bovis was identified by amplifying the RD9 fragment with end-point polymerase chain reaction (PCR). Results: Eight-hundred and fifty tuberculosis-diagnosed patients were included; in 441 cases, Mycobacterium tuberculosis was confirmed by positive culture (250 pulmonary, 65 ganglionic, 39 renal, 34 meningeal, 25 miliary, 14 pleural, 8 peritoneal, 4 bone and 2 pericardial cases). Forty-eight strains (10.8\%) were typified as M. bovis by amplification of the RD9 fragment with end-point PCR. Conclusions: M. bovis is not currently thought of a causative agent of tuberculosis, which could be the cause of pharmacological treatment failure. In this study, the main extrapulmonary form was observed to be cervical lymphadenopathy.
\end{abstract}

KEY WORDS: Tuberculosis. Mycobacterium bovis. End-point polymerase chain reaction.

\section{Identificación de Mycobacterium bovis en pacientes con diagnóstico de tuberculosis pulmonar y extrapulmonar}

\section{Resumen}

Introducción: En México existe un incremento alarmante de casos de infección pulmonar y extrapulmonar por Mycobacterium bovis. La falta de identificación oportuna deriva en complicaciones y eleva la mortalidad. Objetivo: Conocer la frecuencia de infecciones por Mycobacterium bovis en muestras clínicas de pacientes con tuberculosis, identificadas en el laboratorio de micobacterias en un hospital de concentración de la Ciudad de México. Método: Estudio prospectivo, descriptivo. Se estudiaron cepas aisladas de material biológico en cultivos Löwestein-Jensen y MGITI960. La identificación de Mycobacterium bovis se realizó mediante la amplificación del fragmento RD9 por PCR punto final. Resultados: Se incluyeron 850 pacientes con diagnóstico de tuberculosis, en 441 casos se confirmó Mycobacterium tuberculosis por cultivo positivo (250 casos pulmonares, 65 ganglionares, 39 renales, 34 meníngeos, 25 miliares, 14 pleurales, ocho peritoneales, cuatro óseos y dos pericárdicos). Se tipificaron 48 cepas (10.8\%) como Mycobacterium bovis por amplificación del fragmento RD9 por PCR punto final. Conclusiones: Actualmente no se piensa en Mycobacterium bovis como agente causal de tuberculosis, lo que pudiera ser la causa del fracaso del tratamiento farmacológico. En este estudio se observó que la principal forma extrapulmonar es la linfadenopatía cervical.

PALABRAS CLAVE: Tuberculosis. Mycobacterium bovis. PCR punto final.

\footnotetext{
Correspondence:

Date of reception: 17-07-2019

*Alejandro Hernández-Solís

Date of acceptance: 05-09-2019

E-mail: dhernandezsolis@yahoo.com.mx

DOI: 10.24875/GMM.M20000365

Gac Med Mex. 2019;155:564-568

Contents available at PubMed

0016-3813/O 2019 Academia Nacional de Medicina de México, A.C.. Pub license (http://creativecommons.org/licenses/by-nc-nd/4.0/).
} 


\section{Introduction}

Mycobacterium tuberculosis is the main causative agent of tuberculosis in humans, whereas Mycobacterium bovis normally causes tuberculosis in animals. ${ }^{1}$ The term zoonotic tuberculosis is used to describe Mycobacterium bovis infection in humans.

Tuberculosis transmission caused by both pathogens can occur from humans to animals and vice versa. ${ }^{2}$ The World Health Organization points out that, currently, it is a public health problem that is poorly addressed in emerging countries. In recent studies, Mycobacterium bovis and Mycobacterium tuberculosis coinfection has been observed. ${ }^{3}$

The infection is acquired by direct and indirect contact with animals and their secretions, which increases the risk of zoonotic tuberculosis. Known risk factors include working history of the individual, consumption of raw milk and raw dairy products, especially in extrapulmonary tuberculosis cases. ${ }^{3,4}$

Mycobacterium bovis genotyping has been mainly carried out based on molecular patterns (spoligotypes), which are highly useful to know the spread of the disease; unfortunately, it is an expensive study and it is not available in all laboratories of the country. ${ }^{5}$

In Mexico, the control of this disease is carried out by the National Program against Bovine Tuberculosis, which has managed to reduce the prevalence of the disease in most part of the country, except in regions of high milk production. ${ }^{6}$

Lung infections and extrapulmonary presentations are not adequately documented or recorded; they are believed to be responsible for 5 to $10 \%$ of all tuberculosis infections. Not all Mycobacterium bovis-infected individuals develop active disease, although patients with immunosuppression are more prone to infection. 5,6

One of the challenges health personnel is faced with lies in identifying the cause of the disease, given that Mycobacterium bovis and Mycobacterium tuberculosis are clinically and radiologically indistinguishable from each other. The first case is suspected when a patient does not improve with first line treatment, which makes it essential for the species involved to be known in order to establish a specific treatment, since Mycobacterium bovis is naturally resistant to pyrazinamide. ${ }^{7}$

The purpose of this study is to determine the frequency of Mycobacterium bovis in clinical samples of patients with pulmonary and extrapulmonary tuberculosis, processed in the mycobacterial laboratory of a tertiary care specialty hospital, from January 2013 to December 2018.

\section{Method}

A prospective study was designed, where clinical samples of patients admitted with a diagnosis of tuberculosis between January 2013 and December 2018 were used to establish the frequency of Mycobacterium bovis as etiological agent in subjects with pulmonary and extrapulmonary tuberculosis forms at General Hospital of Mexico "Dr. Eduardo Liceaga". Inclusion criteria were: patients older than 18 years in whose clinical samples (sputum, biopsy material, urine, pleural fluid and cerebrospinal fluid) mycobacterial growth was obtained in the Löwestein-Jensen and MGITI960 culture medium, without previous tuberculosis diagnosis or anti-tuberculosis drug treatment.

At hospital admission, every patient diagnosed with tuberculosis underwent history taking, blood cytometry, blood chemistry, human immunodeficiency virus identification by ELISA with confirmation thereof by Western-blot, and chest X-ray.

In extrapulmonary presentations, biopsy was performed (cervical lymphadenopathy, peritoneum, pleura and bone tissue). The obtained samples were fixed in $10 \%$ formalin and embedded in paraffin, sectioned and stained with hematoxylin-eosin for microscopic examination. The tuberculosis diagnosis was established when the findings included granuloma formation with caseous necrosis foci, fibrosis and presence of epithelioid cells.

The biopsy material was taken with aseptic technique and subjected to culture for mycobacteria with Löwestein-Jensen and MGTI960 medium, the gold standard for tuberculosis diagnosis.

Each sample underwent Ziehl-Neelsen staining for AARB, adenosine deaminase (ADA), endpoint polymerase chain reaction (PCR) for the detection of the $1 S 6110$ insertion sequence present in mycobacteria of the Mycobacterium tuberculosis complex, and a skin test with purified protein derivative (PPD-RT23, Statens Seruminstitut, Copenhagen, Denmark) was carried out.

The specimens were homogenized in isotonic saline, buffer phosphate and decontaminated with a $4 \%$ sodium hydroxide solution. After neutralization and centrifugation, each homogenate was re-suspended in distilled water. Acid-alcohol resistant bacilli (AARB) were detected with Ziehl-Neelsen staining. For the cultures, $0.2 \mathrm{~mL}$ of sample homogenates were 
inoculated in bottles with MGIT 960 fluorescent broth (Mycobacterium Growth Indicator Tube, Becton Dickinson, San Jose, CA, USA) and Löwestein-Jensen solid medium. The cultures were incubated at $37{ }^{\circ} \mathrm{C}$ for eight weeks and inspected weekly to assess for growth.

DNA was extracted by thermal shock from a bacterial suspension of each isolate and was used for amplification of approximately $124 \mathrm{bp}$ of the product corresponding to an insertion sequence, IS6110, which is characteristic of the Mycobacterium tuberculosis complex. PCR primers IS6110F (5'-GATCCGCCAGCCCA-GGATCCTGCG-3') and IS6110R (5'-AGGTGCGGACCACCAGCACCTAACC-3') were designed at the Institute of Epidemiological Diagnosis and Reference. The PCR mixture $(50 \mu \mathrm{L})$ was $10 \mathrm{mM}$ Tris- $\mathrm{HCl}, 1.5 \mathrm{mM}$ magnesium chloride, $50 \mathrm{mM}$ potassium chloride (pH 8.3), $1 \mathrm{U}$ taqpolimerase, $2 \mathrm{mM}$ of a dNTP mix (Roche Diagnostics GmbH, Mannheim, Germany), $0.2 \mu \mathrm{M} L$ of DNA from each primer. The reaction conditions were as follows: one 1-minute cycle at $94{ }^{\circ} \mathrm{C}$, followed by 4515 -second cycles at $94{ }^{\circ} \mathrm{C}, 50$ seconds at $70^{\circ} \mathrm{C}$ and one 7 -minute cycle at $72^{\circ} \mathrm{C}$.

For Mycobacterium bovis molecular identification, the DNA preparation was carried out using the boiling method, and it was used as a template for generic PCR of the Mycobacterium complex. To differentiate between Mycobacterium tuberculosis and Mycobacterium bovis, all strains reported as positive for the mycobacteria complex by PCR were analyzed with an RD9 end-point PCR system with three primers (RD9F, RD9R and RD9I), using the zs|2ew method by Samir Das. $^{8}$

To confirm and differentiate Mycobacterium bovis from Mycobacterium tuberculosis, a real-time PCR assay was developed, based on the presence or absence of differentiating regions (RD1, Ext-RD9, RD4, RD9 and RD12) between the genomes of the MTBC members.

\section{Results}

During the study period, 850 patients with suspected tuberculosis were admitted. In 441 cases, Mycobacterium tuberculosis was confirmed by Löwestein-Jensen and MGITI960 positive culture, with an average strain growth of 37 days. Twelve cultures were eliminated due to contamination; $70 \%$ (310) of patients were males and $30 \%$ (131), females; mean age was 41 years. PPD was positive with induration larger than $10 \mathrm{~mm}$ in $25 \%$ of patients.

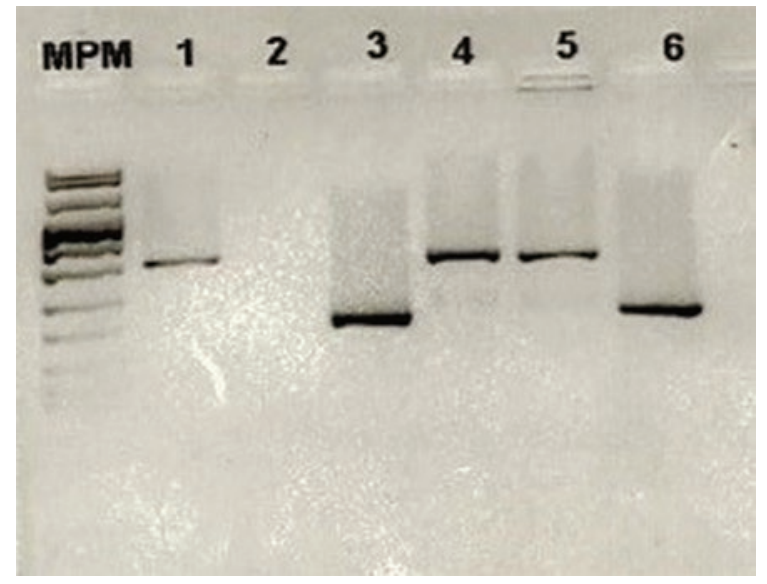

Figure 1. Mycobacterium tuberculosis/Mycobacterium bovis differentiation by end-point PCR. Analysis of the RD9 fragment in $2.5 \%$ agarose gel. MPM, molecular size marker VIII (ROCHE); 1 , Mycobacterium tuberculosis $\mathrm{H} 37 \mathrm{Rv}$ strain; 2, PCR reagents control; 3, Mycobacterium bovis BCG; 4 and 5, samples 3209 and 1687, respectively, identified as Mycobacterium tuberculosis; 6, sample 1916 identified as Mycobacterium bovis.

In $30 \%$ of cases, acquired immunodeficiency syndrome (HIV/AIDS) was identified, in $25 \%$, diabetes mellitus, and in $7 \%$, a history of neoplasms on treatment with chemotherapy; there were five patients with chronic use of corticosteroids.

Predominant symptoms were fever (85\%), cough (82\%), weight loss (78\%), asthenia and adynamia (72\%), dyspnea (60\%) and hemoptysis (20\%).

Of the 441 cases with positive cultures, $250(56.6 \%)$ had pulmonary tuberculosis; 65 (14.7\%), lymph node tuberculosis; 39 (8.8\%), renal tuberculosis; 34 (7.7\%), meningeal tuberculosis; 25 (5.6\%), miliary tuberculosis; 14 (3.1\%), pleural tuberculosis; eight $(1.8 \%)$, peritoneal tuberculosis; four (0.9\%), bone tuberculosis; and two, $(0.4 \%)$ pericardial tuberculosis. In all tuberculosis-diagnosed cases, treatment with isoniazid, rifampicin, pyrazinamide and ethambutol was started, which was strictly supervised. Clinical response was evaluated for one year after diagnosis. In the mycobacteria typing, Mycobacterium tuberculosis was identified in $89.2 \%$.

In the present study, 48 strains (10.8\%) were recorded to be positive for Mycobacterium bovis by RD 9 end-point PCR (Figure 1), all positive for Mycobacterium bovis based on the presence of RD1 and ExtRD9 and on the absence of RD4, RD9 and RD12 markers, which represented $100 \%$ concordance between methods. In this group, $50 \%$ were found to be males and $50 \%$ females. PPD was positive only in seven patients, with an average induration of $8 \mathrm{~mm}$. 


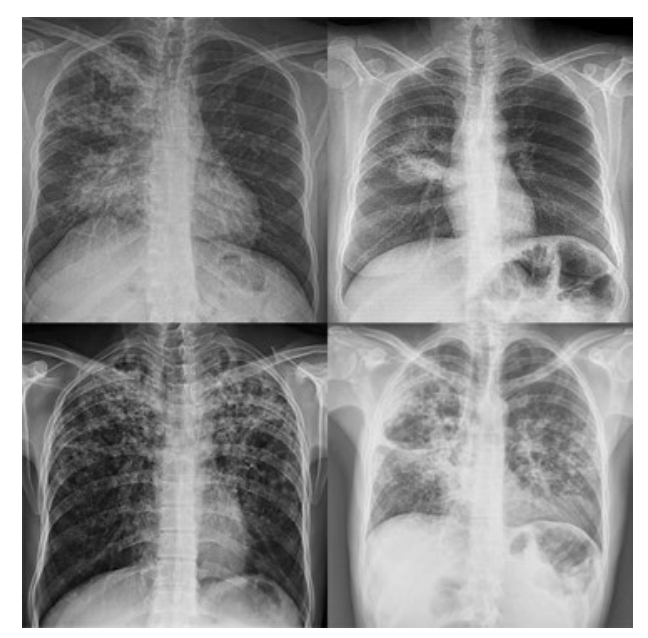

Figure 2. Posteroanterior chest $X$-rays of patients diagnosed with pulmonary tuberculosis by Mycobacterium bovis, where multiple lesions in caverns and alveolar occupation are observed.

By localization, 19 cases (39\%) were lymph node tuberculosis; $10(21 \%)$, pulmonary tuberculosis; nine (19\%), meningeal tuberculosis; seven (14\%), renal tuberculosis; two (4\%), peritoneal tuberculosis; and one $(2 \%)$, pleural tuberculosis. Average growth in the cultures was 31 days, and disease evolution was chronic, with an average of seven years.

\section{Discussion}

Mycobacterium bovis infection in domestic cattle persists in Mexico and represents a public health problem, unlike the countries of Western Europe, which have been declared free of bovine tuberculosis. ${ }^{9}$

In Mexico, the management of Mycobacterium bovis infection is regulated by Mexican Standard NOMZOO-031-1995. In Mexico, the magnitude of the problem of Mycobacterium bovis infection in the general population and in at-risk population is unknown; however, isolated investigations have indicated variations from 2 to $40 \% .^{10}$ In Mexico and Latin America, as well as in other developing countries, the prevalence of Mycobacterium bovis disease is not documented or is underestimated. ${ }^{11}$

Mycobacterium bovis infection has a broader spectrum of the disease, which causes for lung disease to progress to chronic stages, as we observed in our study: in the 10 patients with pulmonary tuberculosis, caverns, bronchiectasis and fibrothorax were identified, with a disease evolution of nine years (Figure 2).12,13

Mycobacterium bovis tuberculosis occurs especially in a large number of domestic and wild mammals, particularly in bovine livestock. According to location, cervical lymphadenopathy predominated in our series (39\%), which is consistent with other investigations reports. Evolution of the condition was four years, and in five patients, first-line treatment failed. ${ }^{13,14}$

Spoligotype pattern comparisons have been carried out in Mexico, and they are consistent with those observed in cattle isolates; therefore, tuberculosis in humans caused by Mycobacterium bovis is derived from strains that circulate in bovine livestock. ${ }^{15}$ SB0120 is the most common spoligotype circulating on several continents, while SB0121 exists in Europe, Africa and America. ${ }^{16}$ The determination of Mycobacterium bovis most common spoligotype is the key to find the source of infection and control and prevent the disease.

The factors associated with higher prevalence of the disease are being an immigrant, Hispanic origin, female gender, use of glucocorticoids, low socioeconomic status and extrapulmonary presentations, which we corroborated in our study: mean age of the cases was 41 years, $45 \%$ of the cases came from rural environments with low economic resources, eight cases had occupational exposure, 10 had HIV/ AIDS with a CD4 count $<100$ cells $/ \mathrm{mL} .{ }^{9,10}$ Unlike the results of other investigations, we did not record frequency differences with regard to patient gender. ${ }^{17}$

In emerging countries, 10 to $20 \%$ of cases of tuberculosis are caused by Mycobacterium bovis; however, this percentage might be underestimated because most laboratories do not routinely perform mycobacterial cultures, and only few have systems to identify Mycobacterium bovis. There are few studies investigating genotypic diversity and drug resistance in Mycobacterium bovis from infections in animals or humans..$^{18}$

In this study, we report the presence of Mycobacterium bovis in $10 \%$ of the strains, which is a relevant result given the clinical repercussions it represents. In none of the 41 cases was Mycobacterium bovis infection suspected. ${ }^{11}$

Current guidelines recommend longer treatment for tuberculosis caused by pyrazinamide-resistant organisms such as Mycobacterium bovis, in order to obtain a negative culture in sputum and a higher cure rate. In general, resistance to pyrazinamide has been reported in $38 \%$ of cases in susceptible groups, with poor prognosis. ${ }^{19}$ In our series, $20 \%$ of patients experienced relapse one year after treatment completion; three patients died four months after diagnosis. ${ }^{20}$ 
In cultures identified as Mycobacterium bovis, a 206-bp amplification product was obtained in the RD9 PCR assay, which correlated in $100 \%$ with the culture, biochemical tests and molecular tests results. Owing to its easiness, specificity and low cost, RD9 PCR is a tool of great utility for Mycobacterium bovis routine diagnosis or confirmation in tertiary care hospitals, unlike spoligotyping, which despite being regarded as an uncomplicated tool that generates reproducible results, is focused on epidemiological studies and large-scale sample processing, in addition to being a high-cost resource. ${ }^{8}$

Currently, it is essential to have new molecular biology methods in tertiary care reference hospitals that demonstrate their efficacy to identify different species of mycobacteria, such as multiplex PCR, which allows Mycobacterium bovis to be differentiated from Mycobacterium tuberculosis in sputum and extrapulmonary samples. ${ }^{20,21}$

Molecular epidemiological studies are needed in medicine and in veterinary fields in order to define the importance of zoonotic tuberculosis in human tuberculosis, to know the route of transmission, the risk factors, the dominant spoligotypes among humans and animals and to understand the phylogeographic relationships of strains in Mexico.

\section{Conclusion}

Currently, Mycobacterium bovis is not thought of as the etiologic agent of pulmonary and extrapulmonary tuberculosis; however, it is important for rapid, sensitive and economic molecular biology diagnostic tests to be performed, in order to be able to start an adequate treatment. Collaboration between veterinary and public health services of the country is required in order to decrease the prevalence of this disease.

\section{Acknowledgements}

To the members of the Diagnostic and Molecular Typing and Pathogen Genome laboratories, for their participation in the present study.

\section{References}

1. Esteban J, Muñoz-Egea MC. mycobacterium bovis and other uncommon members of the Mycobacterium tuberculosis complex. Microbiol Spectr. 2016;4.
2. Olea-Popelka F, Muwonge A, Perera A, Dean AS, Mumford E, Erlacher-Vindel $\mathrm{E}$, et al. Zoonotic tuberculosis in human beings caused by Mycobacterium bovis a call for action. Lancet Infect Dis. 2017;17:1-5.

3. Scott C, Cavanaugh JS, Pratt R, Silk BJ, LoBue P, Moonan PK. Human tuberculosis caused by Mycobacterium bovis in the United States, 20062013. Clin Infect Dis. 2016;63:594-601.

4. Scott C, Cavanaugh JS, Silk BJ, Ershova J, Mazurek GH, LoBue PA, et al. Comparison of Sputum-culture conversion for Mycobacterium bovis and M. tuberculosis. Emerg Infect Dis. 2017;23:456-462.

5. Lombardi G, Botti I, Pacciarini ML, Boniotti MB, Roncarati G, Del Monte P. Five-year surveillance of human tuberculosis caused by Mycobacterium bovis in Bologn, Italy: an underestimated problem. Epidemiol Infect. 2017; 145:3035-3039.

6. Torres-González P, Cervera-Hernández ME, Martínez-Gamboa A, García-García L, Cruz-Hervert LP, Bobadilla-Del Valle M, et al. Human tuberculosis caused by Mycobacterium bovis: a retrospective comparison with Mycobacterium tuberculosis in a Mexican tertiary care centre, 20002015. BMC Infect Dis. 2016;16:657.

7. Kurbatova EV, Cavanaugh JS, Dalton T, Click ES, Cegielski JP. Epidemiology of pyrazinamide-resistant tuberculosis in the United States, 1999-2009. Clin Infect Dis. 2013;57:1081-1093.

8. Das S, Das SC, Verma R. Occurrence of RD9 region and 500 bp fragment among clinical isolates of Mycobacterium tuberculosis and Mycobacterium bovis. Microbiol Immunol. 2007;51:231-234.

9. Sandoval-Azuara SE, Muñiz-Salazar R, Perea-Jacobo R, Robbe-Austerman S, Perera-Ortiz A, López-Valencia G, et.al. Whole genome sequencing of mycobacterium bovis to obtain molecular fingerprints in human and cattle isolates from Baja California, Mexico. Int J Infect Dis. 2017;63:48-56.

10. Zaragoza-Bastida A, Rivero-Pérez N, Valladares-Carranza B, Isaac-Olivé K, Moreno-Pérez P, Sandoval-Trujillo $H$, et al. Molecular identification of mycobacterium species of public health and veterinary importance from cattle in the south state of México. Can J Infect Dis Med Microbiol. 2017;2017:6094587.

11. Müller B, Dürr S, Alonso S, Hattendorf J, Laisse CJ, Parsons SD, van Helden PD, Zinsstag J. Zoonotic mycobacterium bovis-induced tuberculosis in humans. Emerg Infect Dis. 2013;19:899-908.

12. Laniado-Laborín R, Muñiz-Salazar R, García-Ortiz RA, Vargas-Ojeda AC, Villa-Rosas C, Oceguera-Palao L. Molecular characterization of Mycobacterium bovis isolates from patients with tuberculosis in Baja California, Mexico. Infect Genet Evol. 2014;27:1-5

13. Siala M, Smaoui S, Taktak W, Hachicha S, Ghorbel A, Marouane C, et al First-time detection and identification of the Mycobacterium tuberculosis complex members in extrapulmonary tuberculosis clinical samples in south Tunisia by a single tube tetraplex real-time PCR assay. PLoS Negl Trop Dis. 2017:5:e0005572.

14. Cicero R, Olivera H, Hernández SA, Ramírez CE, Escobar GA. Frequency of Mycobacterium bovis as an etiologic agent in extrapulmonary tuberculosis in HIV-positive and -negative Mexican patients. Eur J Clin Microbiol Infect Dis. 2009 May:28:455-460.

15. Perea-Razo CA, Rodríguez-Hernández E, Román-Ponce SI, Milián-Suazo F, Robbe-Austerman S, Stuber T, et al. Molecular epidemiology of cattle tuberculosis in Mexico through whole genome sequencing and spoligotyping. PLoS One. 2018;13:e0201981.

16. Ghavidel M, Mansury D, Nourian K, Ghazvini K. The most common spoligotype of Mycobacterium bovis isolated in the world and the recommended loci for VNTR typing; a systematic review. Microb Pathog. 2018;118:310-315

17. Silva MR, Rocha ADS, Araújo FR, Fonseca-Júnior AA, Alencar AP, Suffys PN, et al. Risk factors for human Mycobacterium bovis infections in an urban area of Brazil. Mem Inst Oswaldo Cruz. 2018; 113:e170445.

18. Moghaddam $\mathrm{R}$, Mosavari $\mathrm{N}$, Mahalati AH. Molecular identification of Mycobacterium tuberculosis complex isolates from KermanshahProvince, Iran. Int J Mycobacteriol. 2016;5:S203.

19. Franco MMJ, Ribeiro MG, Pavan FR, Miyata M, Heinemann MB, De Souza AF, et al. Genotyping and rifampicin and isoniazid resistance in Mycobacterium bovis strains isolated from the lymph nodes of slaughtered cattle. Tuberculosis (Edinb). 2017;104:30-37.

20. Munir S, Mahmood N, Shahid S, Khan ML. Molecular detection of isoniazid, rifampin and ethambutol resistance to $\mathrm{m}$. tuberculosis and $\mathrm{M}$. bovis in multidrug resistant tuberculosis (MDR-TB) patients in Pakistan. Microb Pathog. 2017:110:262-274.

21. Jabbar A, Khan J, Ullah A, Rehman H, Ali I. Detection of mycobacterium tuberculosis and Mycobacterium bovis from human sputum samples through multiplex PCR. Pak J Pharm Sci. 2015;28:1275-1280. 